\title{
A discussion on modeling shape memory alloy embedded in a composite laminate as axial force and elastic foundation
}

\author{
Yin Zhang, Ya-Pu Zhao * \\ State Key Laboratory of Nonlinear Mechanics (LNM), Institute of Mechanics, Chinese Academy of Sciences, Beijing 100080, People's Republic of China
}

Received 16 May 2005; accepted 10 November 2005

Available online 27 December 2005

\begin{abstract}
In this paper, the possible error sources of the composite natural frequencies due to modeling the shape memory alloy (SMA) wire as an axial force or an elastic foundation and anisotropy are discussed. The great benefit of modeling the SMA wire as an axial force and an elastic foundation is that the complex constitutive relation of SMA can be avoided. But as the SMA wire and graphite-epoxy are rigidly bonded together, such constraint causes the re-distribution of the stress in the composite. This, together with anisotropy, which also reduces the structural stiffness can cause the relatively large error between the experimental data and theoretical results.

(C) 2005 Elsevier Ltd. All rights reserved.
\end{abstract}

Keywords: Shape memory alloy; Constitutive relation; Constraint; Stress redistribution

\section{Introduction: the problem}

In Fig. 16 of their paper [1], Epps and Chandra noticed that if the influence of shape memory alloy (SMA) wire is simply modeled as an axial force on the beam, the analysis over-predicts the natural frequency, especially when the temperature is high. The stress inside their SMA wire is tensile, which tends to stiffen the composite structure. Epps and Chandra presented another model which models the SMA wire as an elastic foundation [1]. The elastic foundation property is a function of SMA tension as shown in their appendix [1]. Such elastic foundation modeling dramatically reduce the their computation difference with the experimental data when the temperature is high. While, at low temperature, the two models (axial force and elastic foundation models) hold almost the same relatively large error compared with the experimental data. As it is noticed that the in Epps and Chandra's governing equation (their Eq. (12)) [1], the tensile effect due to SMA wire is only implicitly included in elastic foundation spring constant $k(x)$. Because the SMA wire and the epoxy are bonded, this

\footnotetext{
* Corresponding author. Tel.: +8610 62658008; fax: +861062561284. E-mail address: yzhao@Inm.imech.ac.cn (Y.-P. Zhao).
}

constraint redistributes the stress inside SMA wire, which affects the actual stress distribution in both SMA wire and epoxy layer. As the SMA wire is modeled as the elastic foundation, Eq. (12) in Epps and Chandra's paper [1] actually is the governing equation for the epoxy layer, in which axial force does not show explicitly. The paper aims to discuss such constraint influence on the stress redistribution, which directly affects the computation of the composite structure natural frequencies. Other factor influencing the computation like the anisotropy is also discussed.

The detailed formulation of 1-D and 2-D models of SMA layer/wire embedded in an orthotropic graphite/ epoxy composite matrix layer is presented by Jia and Rogers [2], and Xue and Mei [3]. Their method basically is to apply Hooks Law (constitutive relations) to SMA and composite matrix separately and sum both forces of the SMA layer and composite matrix together to find out the effective Youngs modulus, coefficients of thermal expansion, thus to find out the constitutive relations. The constitutive relations found by this way is widely used in many papers $[1,3,4]$. The very essence of their traditional method is to assume one strain variable in the constitutive relations, which implicitly includes the constraint. Assuming the continuity of strain in the different layer of the compos- 
ite is the traditional method (and as the result, the stress is discontinuous in the different layer). All the stress redistribution due to the constraint is implicitly included in the assumption. In this paper, the traditional method is re-stated, analyzed and compared with the modeling SMA as an axial force or an elastic foundation. Modeling the SMA layer/wire as an axial force or an elastic foundation can avoid the complex constitutive relation for SMA, which reduces the computation effort. However, the potential danger of neglecting such constraint exists. Traditional method of modeling the SMA layer/wire in the composite is strongly suggested for the analysis of the composite to avoid such danger.

\section{Statement and analysis of constitutive relation on the composite material}

The following is a simplified 2-D effective constitutive relation of the composite structure presented by Xue and Mei [3]. Fig. 1 shows the schematic diagram of a composite beam/plate with the SMA layer embedded. SMA has the following constitutive relations:

$\sigma_{\mathrm{s} 1}=E_{\mathrm{s}} \epsilon_{1}+\sigma_{\mathrm{r}} \quad\left(T>T_{\mathrm{s} 1}\right.$, SMA activated $)$,

and

$\sigma_{\mathrm{s} 1}=E_{\mathrm{s}}\left(\epsilon_{1}-\alpha_{\mathrm{s} 1} \Delta T\right) \quad\left(T<T_{\mathrm{s} 1}\right.$, SMA inactivated $)$.

For graphite/epoxy composite matrix material, it is

$\sigma_{\mathrm{m} 1}=E_{\mathrm{m} 1}\left(\epsilon-\alpha_{\mathrm{m} 1} \Delta T\right)$.

$E_{\mathrm{s}}, E_{\mathrm{m} 1}$ are the Young's moduli of SMA and composite matrix material. $\sigma_{\mathrm{r}}$ is the recovery stress and its detailed expression is given in Liang and Roger's paper [5]. In general, $E_{\mathrm{s}}$ and $\sigma_{\mathrm{r}}$ are both temperature dependent. The subscript 1 stands for the direction indicated in Fig. 1.T is the temperature and $T_{\mathrm{s} 1}$ is the temperature when SMA is activated. $\alpha_{\mathrm{s} 1}$ and $\alpha_{\mathrm{m} 1}$ are the coefficients of thermal expansion (CTE) for SMA and composite matrix material in 1 direction, respectively. Thermal effects are generally greater

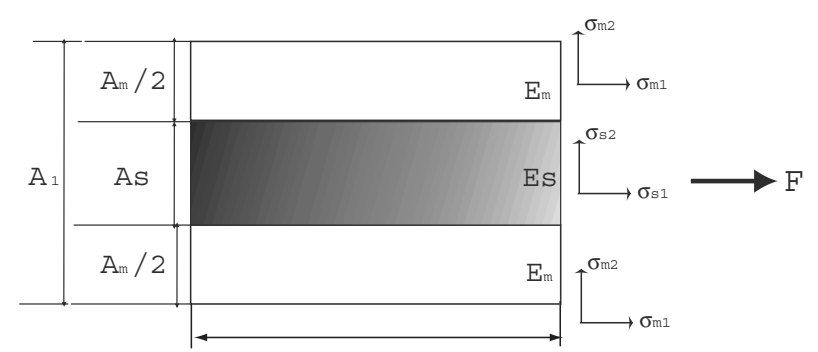

$\mathrm{A}_{2}$

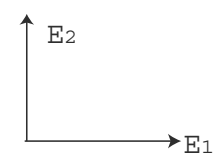

Fig. 1. A schematic diagram of the composite with SMA embedded and its layers' dimensions. The composite is treated as an orthotropic structure. at higher temperature [6]. Thus CTE is temperature dependent in general and in the model above, it is taken as a constant. $\Delta T$ is the composite temperature difference with the ambient environment. $\epsilon_{1}$ and $\epsilon$ stand for the strain of SMA and the strain of the composite matrix in 1 direction, respectively. Here we deliberately write the strains for the SMA and composite matrix as two variables to emphasize the assumption of one strain variable. In Xue and Mei's paper [3], it is only one strain variable for the strains in SMA and composite matrix layer (Eqs. (1), (2) and (4) in their paper). By assuming such one strain variable, the continuity of strain/displacement of SMA and composite matrix at interfaces is guaranteed and obviously the stress is discontinuous at interfaces. From now on, only one strain variable $\epsilon_{1}$ is used in 1 direction. It is worth pointing out that $\epsilon_{1}$ is the total strain [6], which includes mechanical, thermal and recovery ones. The resultant force $F_{1}$ in 1 direction is:

$F_{1}=\sigma_{1} A_{1}=\sigma_{\mathrm{s} 1} A_{\mathrm{s}}+\sigma_{\mathrm{m} 1} A_{\mathrm{m}}$.

$A_{1}$ is the total area in 1 direction, $A_{\mathrm{s}}$ is the SMA layer area and $A_{\mathrm{m}}$ is the composite matrix area. Thus, the effective stress for the whole composite beam is:

$\sigma_{1}=\sigma_{\mathrm{s} 1} V_{\mathrm{s}}+\sigma_{\mathrm{m} 1} V_{\mathrm{m}}$.

Here $V_{\mathrm{s}}$ and $V_{\mathrm{m}}$ are called volume fractions and the following expressions hold for them:

$V_{\mathrm{s}}=\frac{A_{\mathrm{s}}}{A_{1}}, \quad V_{\mathrm{m}}=\frac{A_{\mathrm{m}}}{A_{1}}$.

In the case of $T>T_{\mathrm{s}}$, the constitutive relation (Eq. (5)) can be rewritten as follows:

$$
\begin{aligned}
\sigma_{1} & =\left(E_{\mathrm{s}} \epsilon_{1}+\sigma_{\mathrm{r}}\right) V_{\mathrm{s}}+E_{\mathrm{m} 1}\left(\epsilon_{1}-\alpha_{\mathrm{m} 1} \Delta T\right) V_{\mathrm{m}} \\
& =E_{1} \epsilon_{1}+\sigma_{\mathrm{r}} V_{\mathrm{s}}-E_{\mathrm{m} 1} \alpha_{\mathrm{m} 1} \Delta T V_{\mathrm{m}} .
\end{aligned}
$$

In the case of $T<T_{\mathrm{s}}$, it is

$\sigma_{1}=E_{\mathrm{s}}\left(\epsilon_{1}-\alpha_{\mathrm{s}} \Delta T\right) V_{\mathrm{s}}+E_{\mathrm{m} 1}\left(\epsilon_{1}-\alpha_{\mathrm{m} 1} \Delta T\right) V_{\mathrm{m}}$.

Here $E_{1}$ is the effective Young's modulus for the whole composite structure in 1 direction

$E_{1}=E_{\mathrm{s}} V_{\mathrm{s}}+E_{\mathrm{m} 1} V_{\mathrm{m}}$.

In 2 direction, there is no recovery stress. The following equations hold for SMA and composite matrix in 2 direction [3]:

$\sigma_{\mathrm{s} 2}=E_{\mathrm{s}}\left(\epsilon_{\mathrm{s}}-\alpha_{\mathrm{s}} \Delta T\right)$,

and

$\sigma_{\mathrm{m} 2}=E_{\mathrm{m} 2}\left(\epsilon_{\mathrm{m}}-\alpha_{\mathrm{m} 2} \Delta T\right)$.

Here $\epsilon_{\mathrm{s}}$ and $\epsilon_{\mathrm{m}}$ are the SMA strain and composite matrix strain, respectively. Unlike the case in 1 direction, they are two independent variables in 2 direction. Here SMA is assumed isotropic and composite matrix material is anisotropic. $E_{\mathrm{m} 2}$ and $\alpha_{\mathrm{m} 2}$ are the composite matrix Young's modulus and CTE in 2 direction, respectively. Eqs. (9) and (10) can also be rewritten as

$\epsilon_{\mathrm{s}}=\left(\sigma_{\mathrm{s} 2}+\alpha_{\mathrm{s}} E_{\mathrm{s}} \Delta T\right) / E_{\mathrm{s}}$, 
and

$\epsilon_{\mathrm{m}}=\left(\sigma_{\mathrm{m} 2}+\alpha_{\mathrm{m} 2} E_{\mathrm{m} 2} \Delta T\right) / E_{\mathrm{m} 2}$.

In 2 direction, $\sigma_{2}=\sigma_{\mathrm{s} 2}=\sigma_{\mathrm{m} 2}$, the average strain and stress in 2 direction are found to be

$\epsilon_{2}=\frac{\sigma_{2}\left(V_{\mathrm{s}} E_{\mathrm{m} 2}+V_{\mathrm{m}} E_{\mathrm{s}}\right)}{E_{\mathrm{s}} E_{\mathrm{m} 2}}+\left(\alpha_{\mathrm{m} 2} V_{\mathrm{m}}+\alpha_{\mathrm{s}} V_{\mathrm{s}}\right) \Delta T$,

and

$\sigma_{2}=\frac{E_{\mathrm{s}} E_{\mathrm{m} 2}\left[\epsilon_{2}-\left(\alpha_{\mathrm{m} 2} V_{\mathrm{m}}+\alpha_{\mathrm{s}} V_{\mathrm{s}}\right) \Delta T\right]}{V_{\mathrm{s}} E_{\mathrm{m} 2}+V_{\mathrm{m}} E_{\mathrm{s}}}$.

Therefore, the effective Young's modulus and CTE in 2 direction is

$E_{2}=\frac{E_{\mathrm{s}} E_{\mathrm{m} 2}}{V_{\mathrm{s}} E_{\mathrm{m} 2}+V_{\mathrm{m}} E_{\mathrm{s}}}$,

and

$\alpha_{2}=\alpha_{\mathrm{m} 2} V_{\mathrm{m}}+\alpha_{\mathrm{s}} V_{\mathrm{s}}$.

In 1 direction, the SMA and composite matrix share one strain variable. In the special case of extension in 1 direction alone, they have the same strain/displacement across the whole cross-section. In 2 direction, the SMA and composite matrix share the same loading force/stress. So if SMA and composite layers are treated as springs, the effective moduli of 1 and 2 directions are obtained as the springs are in parallel and serial configurations, respectively. Here the effective moduli $E_{1}$ and $E_{2}$ are actually uniaxial moduli [7]. In the simplified constitutive relations above, Poisson's ratio does not appear and other composite material properties like effective bulk modulus, shear modulus, etc., are not included. For a more general and comprehensive approach of deriving the properties of the composite materials, the reader should refer to Chapter 3 (Effective moduli: cylindrical and lamellar systems) of Christensen's book [7].

The constitutive relations (Eqs. (6), (7) and (14)) do not explicitly show any stress/force between the SMA and composite matrix layer due to the constraint. The constraint in those constitutive relations appear as a simple strain variable, which physically means the interlamellar strain continuity of, i.e., the SMA and composite matrix layer are rigidly bonded. Now we would like to analyze quantitatively the stress/force due to the constraint.

\section{Analysis of a composite bar subject to temperature change}

Here the analysis of a composite bar subject to temperature change is presented. Though the analysis presented includes the thermal effect only, the analysis of composite bar under mechanical loading shares the very essence as that of the thermal analysis. the principle of superposition holds provided that total stresses (thermal, mechanical and of phase transformation) remain within linear elastic limit [8].

In Fig. 2, there are two extension scenarios of a composite bar subject to temperature. Case 1 is the case that the SMA layer and composite matrix layer extend freely without any constraint, and the free extension lengths are $\alpha_{\mathrm{s}} \Delta T L$ and $\alpha_{\mathrm{m} 1} \Delta T L$, respectively. The CTEs of the two materials forming the composite bar are different. When there is a temperature difference with the ambient environment, the material tends to expand by different amount. Under the free extension state, there is no constraint between SMA and composite matrix layer, thus no interlamellar stress/force. As in case 2, the SMA and composite matrix layer are rigidly jointed. In the extension case, they share the same extension length. To compute the extension of case 2, the following two rules are applied [8]:

Rule 1: The compression of SMA + the extension of composite matrix $=$ difference in "free" lengths.

Rule 2: The tensile force applied to the short member by the long member is equal in magnitude to the compressive force applied to the long member by the short member.

Rule 2 can be further translated as the tensile force in composite matrix $=$ the compressive force in SMA. And the difference of "free" lengths in rule 1 is defined as $\alpha_{\mathrm{m} 1} \Delta T L-\alpha_{\mathrm{s}} \Delta T L$. As shown in Fig. 2, rule 1 is the geometric constraint to let the SMA and composite matrix layer have the same extension. Rule 2 here is nothing more than Newton's 3rd law. Mathematically, we translate the two rules as the following two equations:

$$
\begin{aligned}
& \frac{\sigma_{\mathrm{s} 1} L}{E_{\mathrm{s}}}+\frac{\sigma_{\mathrm{m} 1} L}{E_{\mathrm{m} 1}}=\left(\alpha_{\mathrm{m} 1}-\alpha_{\mathrm{s}}\right) \Delta T L, \\
& \sigma_{\mathrm{s} 1} A_{\mathrm{s}}=\sigma_{\mathrm{m} 1} A_{\mathrm{m} 1} .
\end{aligned}
$$

Here $\sigma_{\mathrm{s} 1}$ and $\sigma_{\mathrm{m} 1}$ are the axial stresses of 1 direction in SMA and composite matrix layer, respectively. $E_{\mathrm{s}}, E_{\mathrm{m} 1}$ and $\alpha_{\mathrm{s}}, \alpha_{\mathrm{m} 1}$ are the Young's moduli and CTEs for the two different layers in 1 direction. From the two rules, the exact stress distributions $\left(\sigma_{\mathrm{s} 1}\right.$ and $\left.\sigma_{\mathrm{m} 1}\right)$ in the different layers with the rigidly jointed constraint condition can easily be computed. The SMA here is assumed isotropic. As it is noticed the example above only shows the extension scenario and temperature effect only, the mechanical loading analysis is also presented in Hearn's book [8]. Actually for all the stresses due to phase transformation, mechanical and thermal loading in the extension analysis, the analysis is to model the different layer as the different springs in parallel configuration in 1 direction. In 2 direction, it is the springs in serial configuration. For the analysis of the transverse deflection, rule 1 needs to be adjusted as "the strain/displacement at the layer interface is continuous". The one strain variable approach covers both extension and deflection cases. For the analysis of the transverse deflection, rule 2 is changed as the governing equation. Traditional method of computing neutral axis, using one strain variable to establish the governing for the structure is the best way to enforce rule 1 . 


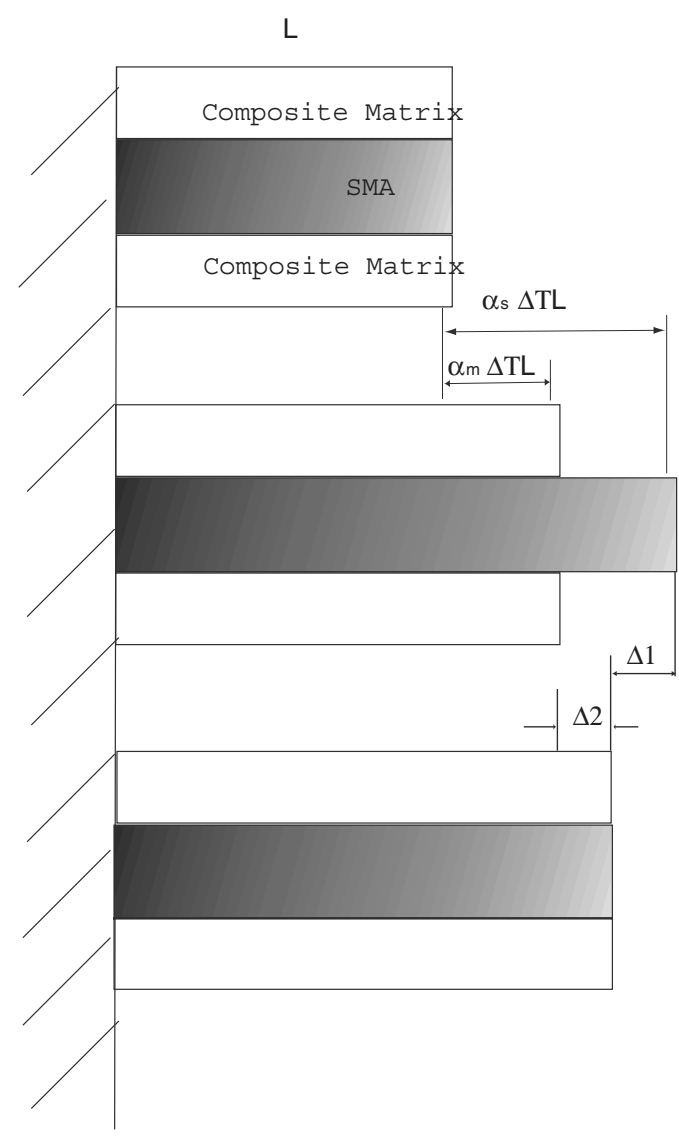

Original Bar without thermal expansion

Case 1: SMA and composite matrix expand independently/freely

Case 2: SMA and composite matrix expand under constraint

$\Delta 1$ : the compression of SMA

$\Delta 2$ : the extension of composite

Fig. 2. The influence of constraint on the composite deformation. Here an elongation example due to thermal expansion is shown. Two scenarios are shown. One is that the composite expands without any constraint, the other is that the composite expands with the constraint which requires all the composite layers expand with the same lengths.

\section{Discussion on the possible error sources on modeling the SMA as axial force and elastic foundation}

Before we discuss the error sources, we would like to present the equation of motion by Epps and Chandra [1], which is used to computed the composite structure natural frequencies. It is their Eq. (12) and has the following expression:

$m \frac{\partial^{2} w}{\partial t^{2}}+\mathrm{EI} \frac{\partial^{4} w}{\partial x^{4}}+k(x) w=0$.

Here $m$ is the beam mass per unit length. $w$ is the beam deflection, EI is the beam bending stiffness. $k$ is the elastic foundation spring constant, it is a function of beam span $(x)$ and SMA tension. Clearly from their equation of motion above, the beam is modeled as Euler-Bernoulli beam.

The first error source is due to the stress redistribution. As shown in the simple analysis of the extension due to thermal expansion, the thermal stress, for example, the SMA layer, cannot be simply determined as $-E \alpha_{\mathrm{s}} \Delta T$. The two rules determine the stress distribution in the extension case. In the transverse vibration, it is quite difficult to show how exact the stress distribution in each layer (it is not constant in each layer as that in the extension case).
But as the constraint of rigid jointing holds, the re-distribution of stress must happen. Mathematically, the use of one strain variable is responsible for this re-distribution. Though Epps and Chandra measure the SMA wire in situ and accurately, the fact of stress re-distribution inside composite matrix layer is not reflected in their governing Eq. (12). As the temperature reaches the phase transformation one, the appearance of recovery stress $\sigma_{\mathrm{r}}$ enhances the stress re-distribution due to the constraint and exacerbates the axial force modeling results as shown in their Fig. 16 [1].

The second one is due to the anisotropy of the composite matrix material. As mentioned above, the constitutive relations derived in (1) and (2) are actually uniaxial type. They are applicable to the uniaxial loading case. For the flexural vibration study, the model adopted by Epps and Chandra [1] is Euler-Bernoulli beam theory for the equivalent homogeneous and isotropic material. As the graphite/ epoxy composite matrix is anisotropic, for such composite structure, it is proper for the whole structure to be treated as an anisotropic beam. As King [9] shows the fact that the beam is restricted to rotate due to the anisotropy, which makes the change of the beam bending stiffness compared with that of the isotropic beam. The anisotropy results in 
some difference of the flexural vibration frequency change as King shows the comparison of the frequencies of anisotropic beam model with those of Timoshenko beam theory [9]. Compared with Euler-Bernoulli beam theory, Timoshenko beam theory considers the strain due to the shear effect and such strain effect can be ignored as the aspect ratio of the thickness to the length is small for isotropic materials. For the "ideal" composite, in which the fiber is inextensible [10], Timoshenko beam theory and anisotropic beam model are suggested even for the composite structure with the small aspect ratio [9]. However, modeling the composite beam as the anisotropic beam or Timoshenko beam dramatically increases the modeling difficulties. Modeling the composite beam structure as Timoshenko beam or anisotropic beam reduces the structural stiffness compared with the Euler-Bernoulli beam theory. Therefore, the natural frequencies decreases and it is noticed that the Euler-Bernoulli beam axial force model by Epps and Chandra [1] overpredicts the natural frequencies.

\section{Summary}

We demonstrate the thermal stress distribution in a composite bar with the rigidly jointed layers due to the temperature effect. The influence of the stress re-distribution due to the constraint on the composite bar extension also holds for the transverse deflection (though in a different way). Due to the constraint that the different layers are rigidly bonded, the stress re-distributes. Measuring one layer stress is not enough to determine the other layers stress states. That is the potential danger of modeling one layer function as an axial force or an elastic foundation while neglecting the other layer stress change due to the constraint. The tradition method of one strain variable, which implicitly includes the constraint effect, is very effective to deal with such problem though it increases the modeling difficulties.

\section{Acknowledgments}

Zhang is thankful to the financial supports from both National Natural Science Foundation of China (NSFC, Grant No. 10502050) and the Scientific Research Foundation for the Returned Overseas Chinese Scholars, State Education Ministry. Zhao is supported by Distinguished Young Scholar Fund of National Natural Science Foundation of China (NSFC, Grant No. 10225209), key project from Chinese Academy of Sciences (Grant No. KJCXSW-L2) and NSFC project (Grant No. 90305020).

\section{References}

[1] Epps J, Chandra R. Shape memory alloy actuation for tuning of composite beams. Smart Mater Struct 1997;20:251-6.

[2] Jia J, Rogers CA. Formulation of a mechanical model of composites laminate with SMA embedded. In: Proceedings at 8th biennial ASME conference on failure prevention and reliability, Montreal (Canada); September, 1989. p. 203-10.

[3] Xue DY, Mei C. A study of the application of shape memory alloy in panel flutter control. In: Proceedings of 5th international conference on recent advances in structural dynamics; 1994. p. 412-22.

[4] Rogers CA, Liang C. Behavior of shape memory alloy reinforced composite plates, Part I: Model of formulations and control concepts. In: Proceedings of the 30th structures, structural dynamics and materials conference, Mobile, AL; 1989. p. 2011-7.

[5] Liang C, Rogers CA. One dimensional thermomechanical constitutive relations for shape memory material. J Intell Mater Struct 1990;20:207-34.

[6] Dowling NE. Mechanical behavior of materials: engineering methods for deformation, fracture, and fatigue. 2nd ed. New Jersey: Prentice Hall; 1999.

[7] Christensen RM. Mechanics of composite materials. Malabar (FL): Krieger Publishing Company; 1991.

[8] Hearn EJ. Mechanics of materials. 2nd ed. Oxford: Pergamon Press; 1985.

[9] King JL. The free transverse vibrations of anisotropic beams. J Sound Vib 1985;98:575-85.

[10] Spencer AJM. Deformation of fibre-reinforced material. Oxford: Clarendon Press; 1972. 